\title{
Morphological and Taxonomic Properties of Tokyovirus, the First Marseilleviridae Member Isolated from Japan
}

\author{
MASAHARU TAKEMURA ${ }^{1 *}$ \\ ${ }^{1}$ Laboratory of Biology, Department of Liberal Arts, Faculty of Science, Tokyo University of Science (RIKADAI), Shinjuku-ku, \\ Tokyo 162-8601, Japan
}

(Received June 28, 2016-Accepted September 20, 2016-Published online November 19, 2016)

Members of the Marseilleviridae family are large DNA viruses with icosahedral particle structures that infect Acanthamoeba cells. The first Marseillevirus to be discovered was isolated in 2009. Since then, several other members of the Marseilleviridae family have been reported, including Lausannevirus, Senegalvirus, Cannes 8 virus, Insectomime virus, Tunisvirus, Melbournevirus, Port-Miou virus, and Brazilian Marseillevirus, which have been isolated from Europe, Africa, Australia, and South America. The morphological and genomic properties of a new Marseilleviridae family member, Tokyovirus, discovered in a water/soil sample from a Japanese river in Tokyo, were described in the present study. Tokyovirus possesses icosahedral particles of up to $200 \mathrm{~nm}$ in diameter, as revealed by a transmission electron microscopy (TEM) analysis, which form a giant virion factory in Acanthamoeba cells. A preliminary genome analysis predicted 487 coding sequences. A dot plot analysis and phylogenetic analysis using family B DNA polymerase, proliferating cell nuclear antigen (PCNA), and DNA-directed RNA polymerase alpha subunit genes revealed that Tokyovirus shares similarities with Marseillevirus, Melbournevirus, and Cannes 8 virus (Marseilleviridae subclade A), but not with Lausannevirus and Port-Miou virus (subclade B), Tunisvirus and Insectomime virus (subclade C), or Brazilian Marseillevirus (subclade D), suggesting that Tokyovirus has evolved separately from the previously described Marseilleviridae members.

Key words: giant virus, Marseilleviridae, virus isolation, phylogenetic analysis, genome analysis

So-called "giant viruses" are generally defined as doublestranded DNA viruses with particle diameters of larger than 200-300 nm, allowing them to be viewed under a light microscope, and a genome longer than $300 \mathrm{kbp}(1)$. The discovery of Acanthamoeba polyphaga mimivirus (APMV), infecting Acanthamoeba cells, in a cooling tower in Bradford (UK) in 2003 revealed the existence of these giant viruses in commonly encountered environments $(15,22)$. These APMV were found to possess icosahedral particles of $750 \mathrm{~nm}$ in diameter and encode DNA genomes of $1.2 \mathrm{Mbp}$, both of which were significantly larger in size than all previously discovered viruses $(15,22)$. The number of APMV continue to expand because studies on these "giant viruses" have revealed the existence of $A$. castellanii mamavirus, A. polyphaga moumouvirus, Cafeteria roenbergensis virus, and Megavirus chilensis, all of which belong to the family Mimiviridae (1, 6, 9, 14, 28). Additionally, studies on "giant viruses" have facilitated the identification of giant viruses of three other types. The first is a group of giant viruses with amphora-shaped particles of approximately $1 \mu \mathrm{m}$ in diameter, which includes the Pandoraviruses (Pandoravirus salinus, $P$. dulcis, and $P$. innopinatum), Pithovirus sibericum, and Mollivirus sibericum $(17,18,20)$. The second type is a recently discovered giant virus lineage, Faustovirus, closely related to Asfarviridae (23). The third is a group of giant viruses with particles smaller than the other Mimiviridae, diameters of $200 \mathrm{~nm}$, and genomes of $300 \mathrm{kbp}$ to $400 \mathrm{kbp}$, and has been named the family Marseilleviridae (10). To date, several DNA viruses of the Marseilleviridae, which were discovered as Marseillevirus

\footnotetext{
* Corresponding author. E-mail: takemura@rs.kagu.tus.ac.jp;
} Tel: +81-3-5228-8373; Fax: +81-3-5228-8373. in a cooling tower in Paris (8), have been reported to reside in a number of locations including rivers, the human gut, and insect bodies. They include Lausannevirus from the Seine River (France), Senegalvirus from an African human gut microbiota, Cannes 8 virus from a cooling tower in Cannes (France), Tunisvirus from fountain water in Tunis (Tunisia), Insectomime virus from insect larvae (Eristalis tenax) in Tunisia, Melbournevirus from a freshwater pond in Melbourne (Australia), Port-Miou virus from a submarine spring in the Cassis Port-Miou Calanque, and Brazilian Marseillevirus from a sewage sample from Brazil (2, 3, 7, 11-13, 16, 27). Marseillevirus-like viruses have also been found in healthy human blood, suggesting that giant viruses are part of the human blood virome (21). However, another research group in the US reported that they were unable to detect Marseilleviruslike viruses in plasma from healthy human blood (19).

This study describes the discovery of a new virus of the family Marseilleviridae, isolated from a water/soil sample from the Arakawa River, located in the eastern area of Tokyo, Japan, which is the first giant virus isolated in Japan. It has been named Tokyovirus, according to the conventional manner to name Marseilleviridae, namely, the city in which they were firstly isolated or analyzed forms their name. Morphological and genome analyses of Tokyovirus and comparisons with other members of the Marseilleviridae reported to date were also performed.

\section{Materials and Methods}

Culture of amoeba cells

A. castellanii (Douglas) Neff (ATCC $30010^{\mathrm{TM}}$ ) cells were purchased from the American Type Culture Collection (ATCC, 
Manassas, VA, USA) and cultured in PYG medium (Proteose peptone, $2 \%[\mathrm{w} / \mathrm{v}]$; yeast extract, $0.1 \%[\mathrm{w} / \mathrm{v}] ; 0.4 \mathrm{mM} \mathrm{CaCl}_{2} ; 4 \mathrm{mM}$ $\mathrm{MgSO}_{4} \cdot 7 \mathrm{H}_{2} \mathrm{O} ; 2.5 \mathrm{mM} \mathrm{Na}_{2} \mathrm{HPO}_{4} \cdot 7 \mathrm{H}_{2} \mathrm{O} ; 2.5 \mathrm{mM} \mathrm{KH} \mathrm{PO}_{4}$; sodium citrate $\cdot 2 \mathrm{H}_{2} \mathrm{O}, 0.1 \%[\mathrm{w} / \mathrm{v}] ; 0.05 \mathrm{mM} \mathrm{Fe}\left(\mathrm{NH}_{4}\right)_{2}\left(\mathrm{SO}_{4}\right)_{2} \cdot 6 \mathrm{H}_{2} \mathrm{O} ; 100 \mathrm{mM}$ glucose; $\mathrm{pH} \mathrm{6.5)}$ at $26^{\circ} \mathrm{C}$ according to the ATCC protocol. In order to avoid contamination of the culture by bacteria and fungi, three antibiotics were added to the culture: $100 \mu \mathrm{g} \mathrm{mL}-1$ of penicillinstreptomycin (GIBCO/Thermo Fischer Scientific, Yokohama, Japan), $100 \mu \mathrm{g} \mathrm{mL} L^{-1}$ of ampicillin (Wako Chemicals USA, Richmond, VA, USA), and $5 \mu \mathrm{g} \mathrm{mL}^{-1}$ of amphotericin B (GIBCO) (20).

\section{Virus isolation}

A water/soil sample was collected from the Arakawa River, located in the eastern area of Tokyo, Japan $\left(35^{\circ} 41^{\prime} 54.21^{\prime \prime} \mathrm{N}\right.$, $\left.139^{\circ} 51^{\prime} 18.41^{\prime \prime} \mathrm{E}\right)$. After the removal of mud by filtration through filter paper with a pore size of $20 \mu \mathrm{m}$ (43; Whatman International, Maidstone, UK), the sample was further filtered through filter paper with a pore size of $0.8 \mu \mathrm{m}$ (Millex-AA; Merck Millipore, Darmstadt, Germany). The filtered sample was concentrated by polyethylene glycol (PEG) precipitation overnight at $4{ }^{\circ} \mathrm{C}$ (final concentration: PEG $10,000,8 \%[\mathrm{w} / \mathrm{v}] ; \mathrm{NaCl}, 0.48 \%$ [w/v]), followed by centrifugation at $1,500 \times \mathrm{g}$ at $4^{\circ} \mathrm{C}$ for $30 \mathrm{~min}(20)$. After removal of the supernatant, the pellet (invisible) was resuspended in $4 \mathrm{~mL}$ of PYG, and filtered again through filter paper with a pore size of $0.8 \mu \mathrm{m}$ (Millex-AA; Merck Millipore). Four milliliters of fresh PYG and $1 \mathrm{~mL}$ of an amoeba cell suspension were added to this viral solution, and the solution was divided and cultured into 56 wells on a 96-well culture plate at $26^{\circ} \mathrm{C}$. After $10 \mathrm{~d}$, amoeba cells were detected in only one of the 56 wells. These cells showed delayed proliferation, and were almost round in shape. The culture supernatant from this one well was inoculated into fresh amoeba cells in three wells of a 96-well culture plate. After 1 week, almost all cells appeared to be round in shape. The supernatant was then inoculated into fresh amoeba cells in one well of a 12-well culture plate. After $4 \mathrm{~d}$, almost all cells appeared to be round in shape. The supernatant was inoculated into fresh amoeba cells in a $25-\mathrm{cm}^{2}$ culture flask. After $2 \mathrm{~d}$, rounded amoeba cells were harvested for observations by transmission electron microscopy. The supernatant was stored at $4^{\circ} \mathrm{C}$ as an isolated virus solution.

\section{Electron microscopic observation}

Harvested cells infected by Tokyovirus were washed twice with PBS, fixed with $2 \%$ glutaraldehyde solution at $4^{\circ} \mathrm{C}$ overnight, and then transferred to fresh $2 \%$ glutaraldehyde solution. Fixed cells were washed three times with PBS and then stained with $2 \%$ osmium tetroxide at $4^{\circ} \mathrm{C}$ for $1 \mathrm{~h}$. Osmium-stained cells were dehydrated in increasing ethanol concentrations $(50 \%, 70 \%, 80 \%, 90 \%, 95 \%$, and $100 \%$ ), each at room temperature for $5 \mathrm{~min}$, and embedded in Epon812 (TAAB Laboratory Equipment, Berks, UK). The hardening of Epon-812 took $2 \mathrm{~d}$ at $60^{\circ} \mathrm{C}$. Ultra-thin sections of $80 \mathrm{~nm}$ were obtained using a microtome (Leica Microsystems, Tokyo, Japan), and were stained with $2 \%$ uranyl acetate for $10 \mathrm{~min}$, followed by lead citrate for $5 \mathrm{~min}$. Observations were performed using TEM (JEM-1400; JEOL, Tokyo, Japan, or H-7600; Hitachi, Tokyo, Japan).

\section{Visualization of virion factories (VF)}

Cultured amoeba cells on a coverslip in a 12-well microplate infected by Tokyovirus at $8 \mathrm{~h}$ post-infection (p.i.) were washed twice with PBS, and fixed with methanol at room temperature for $10 \mathrm{~min}$. Fixed cells on a coverslip were washed twice with PBS, and then completely air-dried. Dried cells were incubated with $500 \mathrm{ng} \mathrm{mL}^{-1}$ of DAPI for $50 \mathrm{~s}$ and then immediately washed twice with PBS. Stained cells on the coverslip were mounted in Vectashield (Vector Laboratories, Burlingame, CA, USA) and visualized using a fluorescence microscope (BX50; Olympus, Tokyo, Japan).

\section{Virus cloning}

Virus cloning was performed according to a cloning method used for Mollivirus (18), with several modifications as described below.
Amoeba cells were seeded into three wells in a 12-well culture plate with $1 \mathrm{~mL}$ of PYG. A total of $0.5 \mathrm{~mL}$ of the isolated virus solution was added to each well, and $1 \mathrm{~h}$ after the inoculation, excess viruses were removed. The cells were washed three times with $1 \mathrm{~mL}$ of PYG, then harvested by scraping. Three serial dilutions were performed in the next nine wells by mixing $100 \mu \mathrm{L}$ of the previous well with $100 \mu \mathrm{L}$ of fresh PYG. The last three dilutions in each case were observed by light microscopy to verify that there were fewer than two amoeba cells in each well. Observations revealed that there were 9,8 , and 2 amoeba cells in each well. Hundreds of fresh amoeba cells were added to the well containing only two cells and were cultured for $3 \mathrm{~d}$ until almost all cells became rounded. The viral clone obtained was then amplified and stored for later use, and genomic DNA extraction was performed as described below.

\section{Genome analysis}

After virus cloning, the genomic DNA of Tokyovirus $(1.1 \mu \mathrm{g})$ was prepared from PYG culture media containing viral particles according to the manufacturer's protocol (NucleoSpin ${ }^{\circledR}$ Tissue; Macherey-Nagel $\mathrm{GmbH}$ and $\mathrm{Co}$. KG). A DNA library for sequencing was prepared and sequencing was performed as described previously (25). A DNA library for sequencing was prepared using a TruSeq Nano DNA LT library prep kit (Illumina, San Diego, CA, USA), and sequencing was performed on a HiSeq 2500 platform (Illumina). The total number of reads was 49,062,650 (each of the reads had a length of 100 nucleotides). Edena software was used for the assembly of $1,000,000$ reads into 68 contigs. Contigs had an average length of 5,481 nucleotides, and the maximum contig had a length of 360,777 nucleotides. The total length of the 68 contigs was 372,707 nucleotides (25). The mapping of reads was performed using the software GeneData Expressionist for Genomic Profiling version 9.1.4a and BWA-MEM, according to the manufacturer's protocol, and was visualized and confirmed using the software Integrative Genomic Viewer (Broad Institute, Cambridge, MA, USA). The prediction of gene function was conducted using NCBI blastp in the NCBI nr and NCBI COG databases. A prediction of the coding region of the Tokyovirus genome was conducted using CRITERIA version $1.05 \mathrm{~b}$ and Glimmer 2 version 2.10 . A prediction of tRNA was conducted using tRNAScan-SE version 1.23, according to the manufacturer's protocol (25). A genome analysis including dot plots for genome comparisons and a Venn diagram to compare gene contents were performed using IMC genomics software (In Silico Biology, Yokohama, Japan).

\section{Phylogenetic analysis}

The amino acid sequences of the B-family DNA polymerase of eight Marseilleviridae family members and APMV were obtained from NCBI protein sequence databases (http://www.ncbi.nlm.nih. gov/). Accession numbers for all sequence data used for this study are shown in Table S1. Sequences were aligned using the ClustalW program implemented in the MEGA7 software (ver. 7.0.14) (26) with default parameters. A maximum-likelihood inference program and the LG model was used as a substitution model with discrete gamma-distributed rate variations and a proportion of sites being invariant (24) to construct the phylogenetic tree. In order to estimate branch support, 100 bootstrap replications were performed. In another analysis, a phylogenetic tree was reconstructed using 30 B-family DNA polymerases, for example from Mimiviridae, Poxviridae, and Pandoraviruses, as described above (Table S1). Additionally, the amino acid sequences of the proliferating cell nuclear antigen (PCNA) and DNA-directed RNA polymerase alpha subunit of nine Marseilleviridae family members and APMV were obtained from NCBI protein sequence databases (Table S2 and S3), and a phylogenetic tree was constructed as described above.

\section{Sequence data}

The almost complete genomic sequence of Tokyovirus has been deposited in DDBJ/ENA/GenBank under accession number AP017398 (25). 


\section{Results and Discussion}

\section{Morphological features of Tokyovirus}

Giant viruses in the narrow sense contain several groups: Mimiviridae, Marseilleviridae, Faustovirus, and the amphoralike giant viruses Pandoravirus, Pithovirus, and Mollivirus (4). The particle sizes of these giant viruses are widely diversified, from Marseilleviridae (approximately $200 \mathrm{~nm}$ in diameter) to Pithovirus (1.5 $\mu \mathrm{m}$ in diameter). Tokyovirus particles were isolated from a muddy freshwater sample from Arakawa River, located in east Tokyo. Arakawa River is one branch of the Tonegawa River, which runs through the largest valley in Japan. The amplification of Tokyovirus and the rounding of all amoeba cells took 2-3 weeks after the inoculation of amoeba cells. This result indicated that Tokyovirus particles were not numerous in the water/soil samples tested. However, it is possible that Tokyovirus is widely distributed throughout Arakawa River and Tonegawa valley. Moreover, other unknown Marseilleviridae family members may inhabit widely separated rivers in Japan.

Rounded cells were subjected to an electron microscopic analysis, which revealed that many Marseillevirus-like particles were present in amoeba cytoplasmic vacuoles. Observations by TEM of infected amoeba cells at $8 \mathrm{~h}$ p.i. revealed intracellular icosahedral particles of approximately $200 \mathrm{~nm}$ in diameter, similar to those of Marseilleviridae such as Marseillevirus and Melbournevirus, with no surrounding fibrils, similar to APMV and Megavirus particles. Tokyovirus particles accumulated in intracytoplasmic vacuoles as with other Marseilleviridae, including putative mature particles (Fig. 1A and B). The main cytopathic effects caused by Tokyovirus against amoeba cells were the rounding of cells and suspension in culture media. The prominent destruction of cells, such as that caused by APMV, was not observed. Tokyovirus infection was accompanied by the large-scale development of VF in the amoeba cytoplasm, similar to that observed with other Marseilleviridae members $(8,10)$. A recent study reported that Marseillevirus possesses unique mechanisms for entry into Acanthamoeba cells, forming giant infectious vesicles surrounded by membranes, which differs from the entry mechanisms of other giant viruses (5). Tokyovirus was also found to display several morphological types in Acanthamoeba cells, such as giant vesicles including many viral particles surrounded by membranes and single particles in the amoeba cytoplasm (Fig. $\mathrm{S} 1)$. These results suggest that this unique entry mechanism into host cells is a universal characteristic of Marseilleviridae.

Observations by TEM of the VF of Tokyovirus at $8 \mathrm{~h}$ p.i. revealed its detailed morphological features. As for Marseillevirus, no remarkable membranous structures were observed at the periphery of the VF (Fig. 1C). The typical morphology of the VF consisted of two clearly divided areas: one in which mature viral particles were abundantly present and the other in which fewer viral particles were present proceeding construction. In the latter area, capsid assembly and DNA encapsidation both proceeded simultaneously (Fig. S2), as observed for Marseillevirus (8). DAPI staining of a $\mathrm{VF}$ from an infected amoeba cell at $8 \mathrm{~h}$ p.i. revealed that it was larger than the amoeba cell nucleus, in many cases occupying $1 / 3$ of the amoeba cytoplasm (Fig. 1C, 2, S3, Table S4 and S5), as observed for other Marseilleviridae members $(5,8)$.
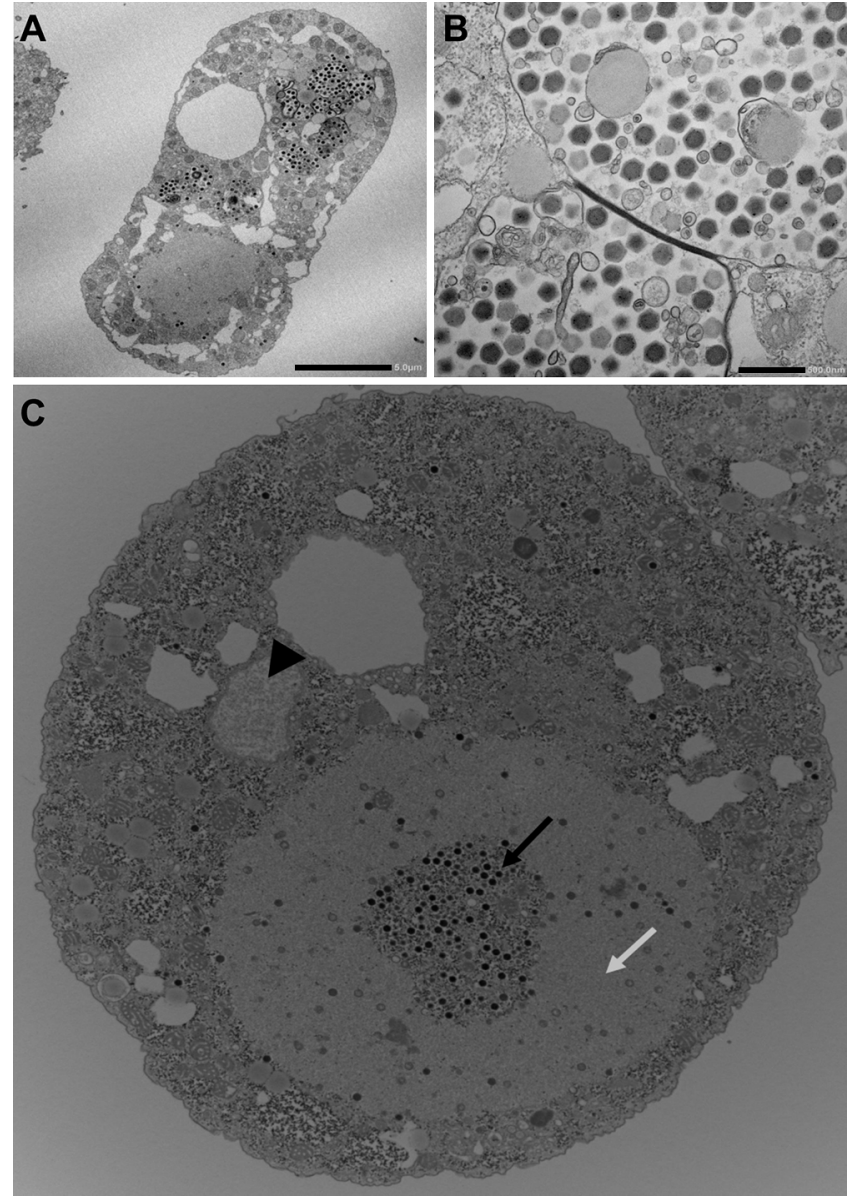

Fig. 1. Transmission electron microscopy images of ultrathin sections of Tokyovirus-infected Acanthamoeba castellanii cells. (A) Comprehensive image of a Tokyovirus-infected amoeba cell at $8 \mathrm{~h}$ p.i. Mature virions (small black particles) are encapsulated in several intracytoplasmic vacuoles. A putative virion factory is seen in the lower-left portion of the cell. The cell nucleus is not evident in this section. Scale bar, $5 \mu \mathrm{m}$. (B) Enlarged image of Tokyoviruses in the intracytoplasmic vacuoles of the amoeba. The particle diameter is approximately $200 \mathrm{~nm}$. Scale bar, $500 \mathrm{~nm}$. (C) Transmission electron microscopy image of a typical virion factory in the cytoplasmic region of Tokyovirus-infected amoeba cells. Virion factories are distinctly classified into two portions: one in which mature virions densely co-exist (black arrow), and another portion surrounding the former (white arrow). In this section, a small part of the cell nucleus is shown (black arrowhead).

\section{Genome analysis}

Tokyovirus has a 360-370 kb genome, of which more than 372,707 bp corresponds to the total contig length (25). A genome analysis using the Illumina HiSeq system showed that the maximum contig was 360,777 nucleotides in length as described above, and was located from 8,609 to 369,355 in deposited genome data under accession number AP017398. There are 43 contigs located from 1 to 8,608 , and 24 contigs located from 369,356 to 372,707 ; therefore, the sequences of these terminal regions have not yet been solved. Nevertheless, this size of the total contig length $(372,707)$ is roughly equivalent to the genome size of other Marseilleviridae family members, such as Marseillevirus $(368,454)$, Cannes 8 virus (374,041), Melbournevirus (369,360), and Lausannevirus $(346,754)(2,8,12,27)$. This nearly complete genome sequence was predicted to contain 487 coding sequences 

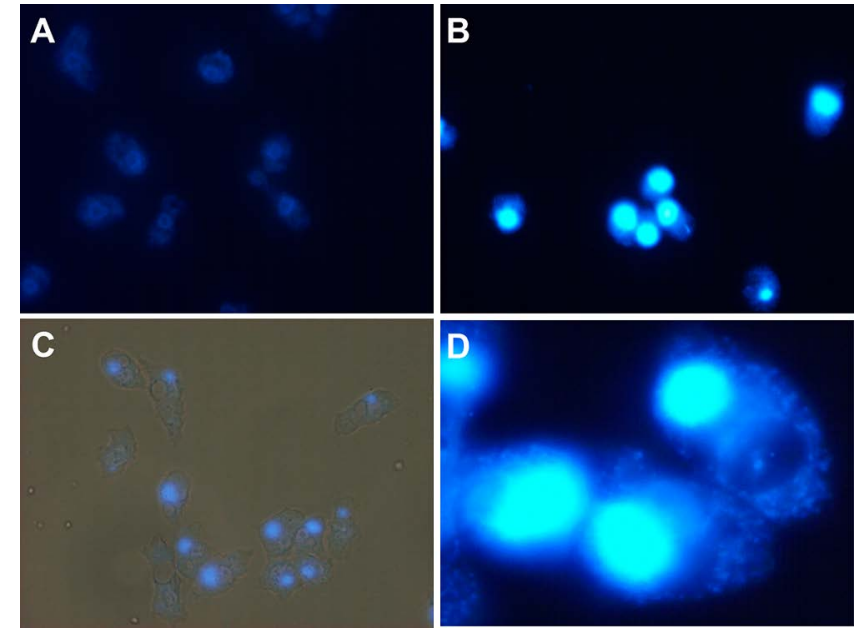

Fig. 2. Fluorescent microscopy images of Tokyovirus-infected Acanthamoeba castellanii cells stained with DAPI. Staining by DAPI was performed at $8 \mathrm{~h}$ p.i., as described in the Materials and Methods. (A) Control amoeba cells without Tokyovirus infection. Cell nuclei were slightly stained and appeared donut-shaped. (B) Tokyovirus-infected amoeba cells. Virion factories were brightly stained. (C) Merged view of fluorescent and phase-contrast images. (D) Enlarged image of Tokyovirusinfected amoeba cells for which the virion factory is brightly stained.

(CDSs) including genes for the 3Rs (replication, recombination, and repair), transcription, amino acid transport and metabolism, histones, and two tRNA genes (one of which is a pseudogene) (25). For example, histone H2A (fused to H2B) and H3-like genes were respectively found on the Tokyovirus genome, similar to other Marseilleviridae. Several translationrelated genes such as translation initiation factor and translation elongation factor genes were also found on the Tokyovirus genome, similar to Cannes 8 virus and Melbournevirus. One CDS was more similar to an unknown CDS of $M$. sibericum than that of other Marseilleviridae, and another was similar with an unknown CDS of $P$. dulcis, similar to other Marseilleviridae. Most CDSs exhibited high homology with other Marseilleviridae family members. On the other hand, several putative CDSs were found to be Tokyovirus-specific CDSs, namely, they did not exist on other Marseilleviridae genomes, which was revealed by a blastp search. The functions of these putative CDSs specific for Tokyovirus have not yet been elucidated.

Dot plots to compare Tokyovirus with seven other Marseilleviridae family members indicated that the Tokyovirus genome was more similar to Marseillevirus, Melbournevirus, and Cannes 8 virus than to Lausannevirus, Tunisvirus, Insectomime virus, and Brazilian Marseillevirus (Fig. 3). The Marseilleviridae family has been divided into four subclades: A, B, C, and D $(2,10,11,13)$. Subclade A includes Marseillevirus, Cannes 8 virus, Senegalvirus, and Melbournevirus. Subclade B includes Lausannevirus and Port-Miou virus. Subclade C includes Tunisvirus and Insectomime virus. Subclade D includes the newly-found Brazilian Marseillevirus. A dot plot analysis suggested that Tokyovirus was similar to subclade A viruses, such as Marseillevirus, Melbournevirus, and Cannes 8 virus because the central part of the Tokyovirus genome showed greater collinearity with these subclade A Marseilleviridae genomes (Fig. 3A, B, and C) than with subclade B Lausannevirus (Fig. 3D), subclade C Tunisvirus and Insectomime virus (Fig. $3 \mathrm{E}$ and F), and subclade D Brazilian Marseillevirus (Fig. 3G). These genomic differences revealed by dot plot analyses between Tokyovirus and other Marseilleviridae are similar to those between previously reported Marseilleviridae of one subclade and that of other subclades (Fig. S4). On the other hand, between Marseilleviridae viruses, both of which were classified into the same subclade, a dot plot analysis hardly detected their differences (Fig. S5), as described previously (12), except for the case of subclade C (Fig. S5E).

It is of particular interest that a part of the Tokyovirus genome is inverted relative to that in subclade A Marseilleviridae genomes (Fig. 3A, B, and C). The region spanning approximately 40,000-90,000 bp of the Tokyovirus genome corresponded to the inverted region positioned around 320,000370,000 of subclade A Marseilleviridae genomes. These results suggest that the genomic composition of Tokyovirus has evolved differently from Marseillevirus, Melbournevirus, and Cannes 8 virus. Although only 1 isolate of Tokyovirus has been obtained to date, Tokyovirus needs to be classified into a new subclade "E", similar to the recently isolated Brazilian Marseillevirus, which is solely classified into subclade D (11).

Venn diagrams showed that Tokyovirus possessed more CDSs with $>90 \%$ sequence identity to those of Cannes 8 virus (63 CDSs), Marseillevirus (47 CDSs), and Melbournevirus (55 CDSs), than to Brazilian Marseillevirus (7 CDSs), Lausannevirus (6 CDSs), and Tunisvirus (4 CDSs) (Fig. 4). The number of CDSs of Tokyovirus with $>90 \%$ sequence identity to Marseillevirus and Melbournevirus was less than that of Marseillevirus and Melbournevirus (Fig. 4). This result supports the suggested classification of Tokyovirus into a new subclade (Fig. 4).

\section{Phylogenetic analysis}

In all of the unrooted phylogenetic trees based on the genes of B-family DNA polymerase, PCNA, and DNA-directed RNA polymerase subunit alpha, Marseilleviridae were grouped into five subclades. Four of the five subclades corresponded to subclade A (Marseillevirus, Melbournevirus, and Cannes 8 virus), subclade B (Lausannevirus and Port-Miou virus), subclade $\mathrm{C}$ (Tunisvirus and Insectomime virus), and subclade D (Brazilian Marseillevirus) (Fig. 5 and S6). A phylogenetic analysis based on the B-family DNA polymerase gene revealed that Tokyovirus is more closely related to Melbournevirus and Cannes 8 virus, both of which belong to subclade A, than to Lausannevirus, Tunisvirus, and Insectomime virus (Fig. 5A and S6). Other phylogenetic analyses using PCNA and DNA-directed RNA polymerase subunit alpha genes also confirmed the putative classification of Tokyovirus described above (Fig. 5B and C). These results indicate that Tokyovirus needs to be classified into a new subclade of Marseilleviridae, which is closer to subclade A than to the other subclades.

\section{Conclusion}

Mature Tokyoviruses in Acanthamoeba cells have icosahedral particles of approximately $200 \mathrm{~nm}$ in diameter and a genome size of $370-380 \mathrm{~kb}$, as reported previously for other 

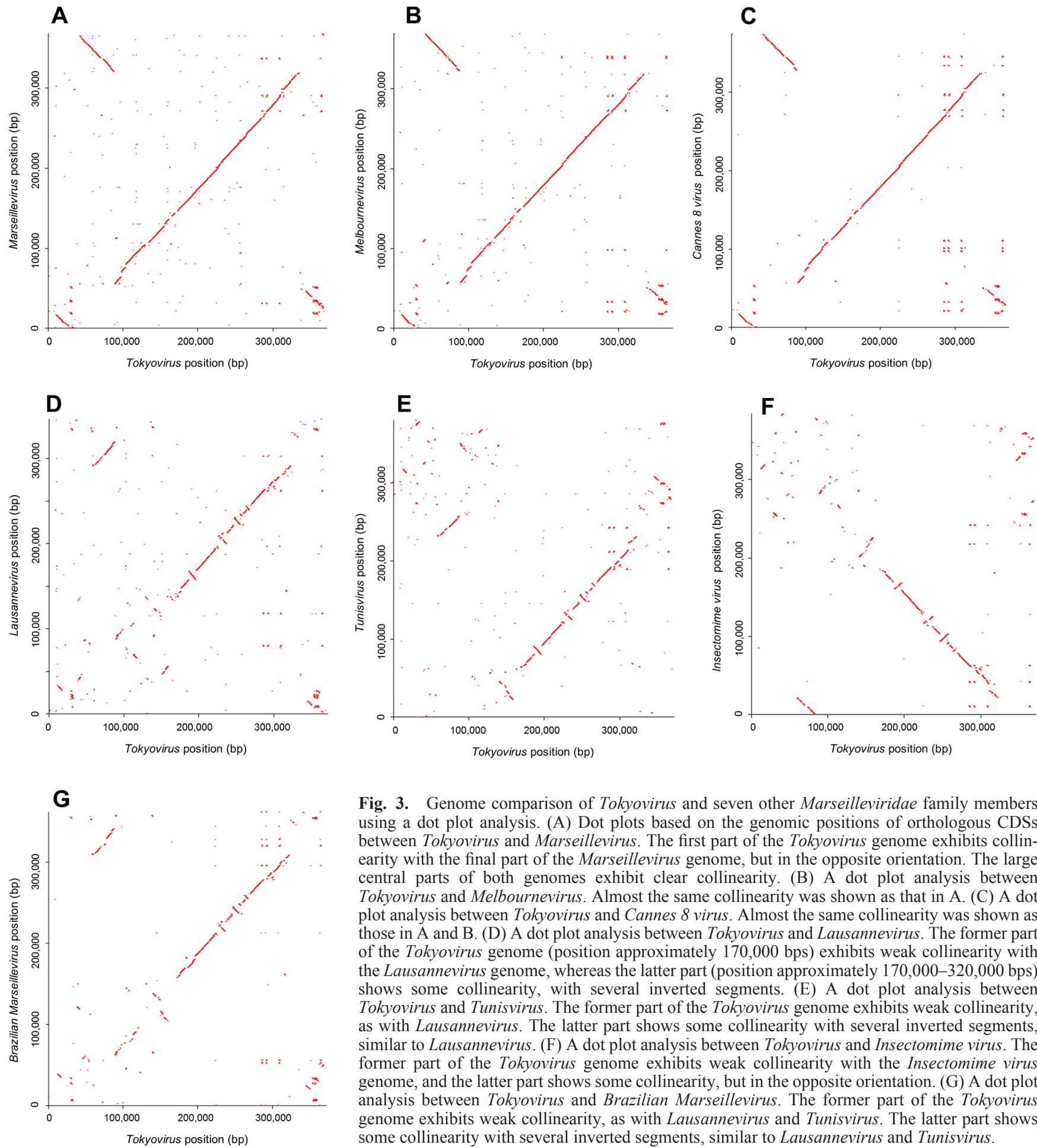

Fig. 3. Genome comparison of Tokyovirus and seven other Marseilleviridae family members using a dot plot analysis. (A) Dot plots based on the genomic positions of orthologous CDSs between Tokyovirus and Marseillevirus. The first part of the Tokyovirus genome exhibits collinearity with the final part of the Marseillevirus genome, but in the opposite orientation. The large central parts of both genomes exhibit clear collinearity. (B) A dot plot analysis between Tokyovirus and Melbournevirus. Almost the same collinearity was shown as that in A. (C) A dot plot analysis between Tokyovirus and Cannes 8 virus. Almost the same collinearity was shown as those in A and B. (D) A dot plot analysis between Tokyovirus and Lausannevirus. The former part of the Tokyovirus genome (position approximately 170,000 bps) exhibits weak collinearity with the Lausannevirus genome, whereas the latter part (position approximately $170,000-320,000 \mathrm{bps}$ ) shows some collinearity, with several inverted segments. (E) A dot plot analysis between Tokyovirus and Tunisvirus. The former part of the Tokyovirus genome exhibits weak collinearity, as with Lausannevirus. The latter part shows some collinearity with several inverted segments, similar to Lausannevirus. (F) A dot plot analysis between Tokyovirus and Insectomime virus. The former part of the Tokyovirus genome exhibits weak collinearity with the Insectomime virus genome, and the latter part shows some collinearity, but in the opposite orientation. (G) A dot plot analysis between Tokyovirus and Brazilian Marseillevirus. The former part of the Tokyovirus genome exhibits weak collinearity, as with Lausannevirus and Tunisvirus. The latter part shows some collinearity with several inverted segments, similar to Lausannevirus and Tunisvirus.

Marseilleviridae family members. The VF of Tokyovirus become enlarged, in many cases occupying $1 / 3$ of the amoeba cytoplasm. In these VF, densely stained regions are evident, within which abundant mature particles are visible. These characteristics are typical of Marseilleviridae family members. According to the genome analysis, Tokyovirus is closely related to subclade A of Marseilleviridae; however, its classification into a new subclade "E" is suggested. The mecha- nisms by which Tokyovirus evolved away from other Marseilleviridae family members have not yet been elucidated. Ecological studies on Marseilleviridae, isolated from various environments, are of important significance for clarifying the relationship between giant viruses and living organisms, including humans. The results of the present study may stimulate further studies into the worldwide distribution of these viruses and their functional significance. 


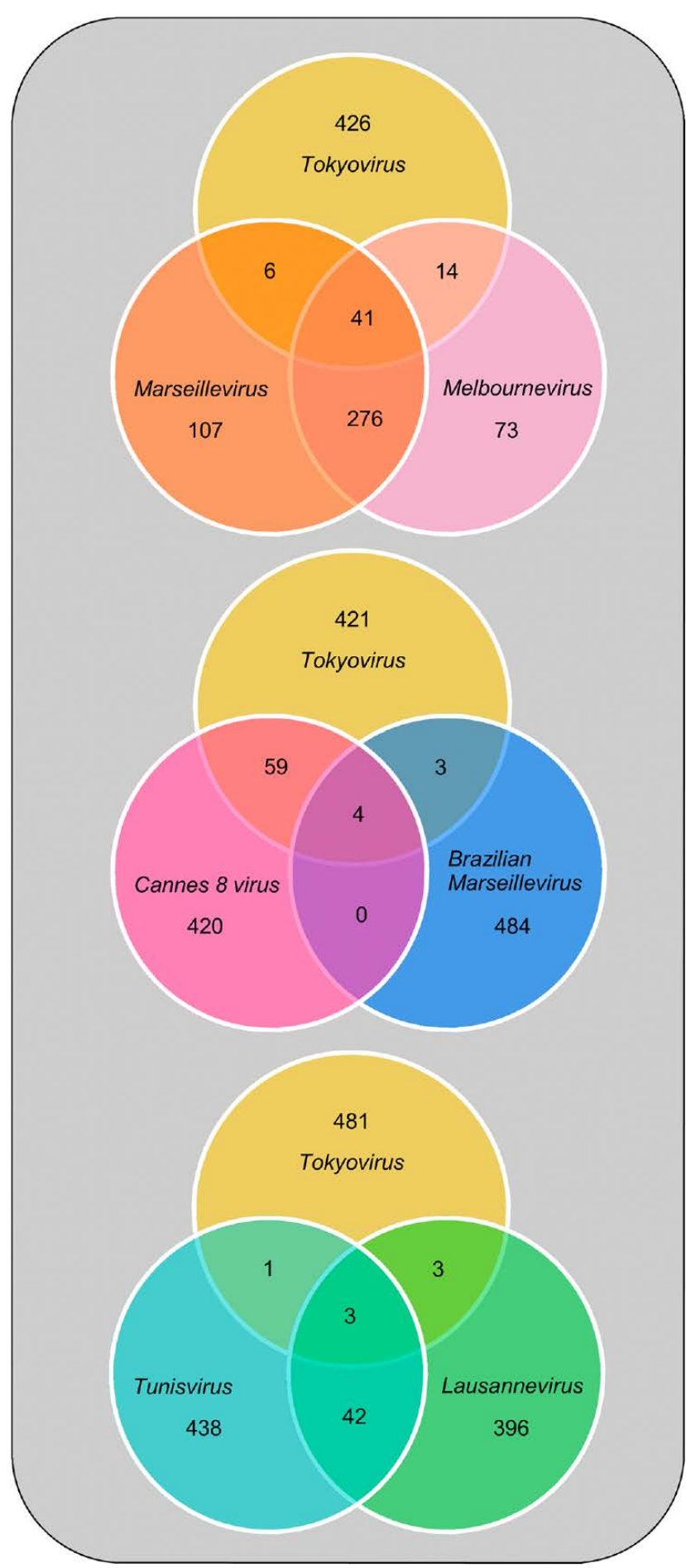

Fig. 4. Venn diagram showing a comparative analysis of gene contents of Tokyovirus with six other Marseilleviridae family members. The numbers of genes showing $>90 \%$ similarity are shown in the merged regions. The upper diagram shows an analysis of Tokyovirus, Marseillevirus, and Melbournevirus. The middle diagram shows an analysis of Tokyovirus, Cannes 8 virus, and Brazilian Marseillevirus. The lower diagram shows an analysis of Tokyovirus, Tunisvirus, and Lausannevirus.

\section{Acknowledgements}

I would like to thank Yasunobu Terabayashi of Takara Bio (Yokkaichi, Mie, Japan) for his technical assistance with the Tokyovirus genome analysis, Koji Itakura of Nagoya University School of Medicine, and members of Hanaichi UltraStructure Research Institute for their
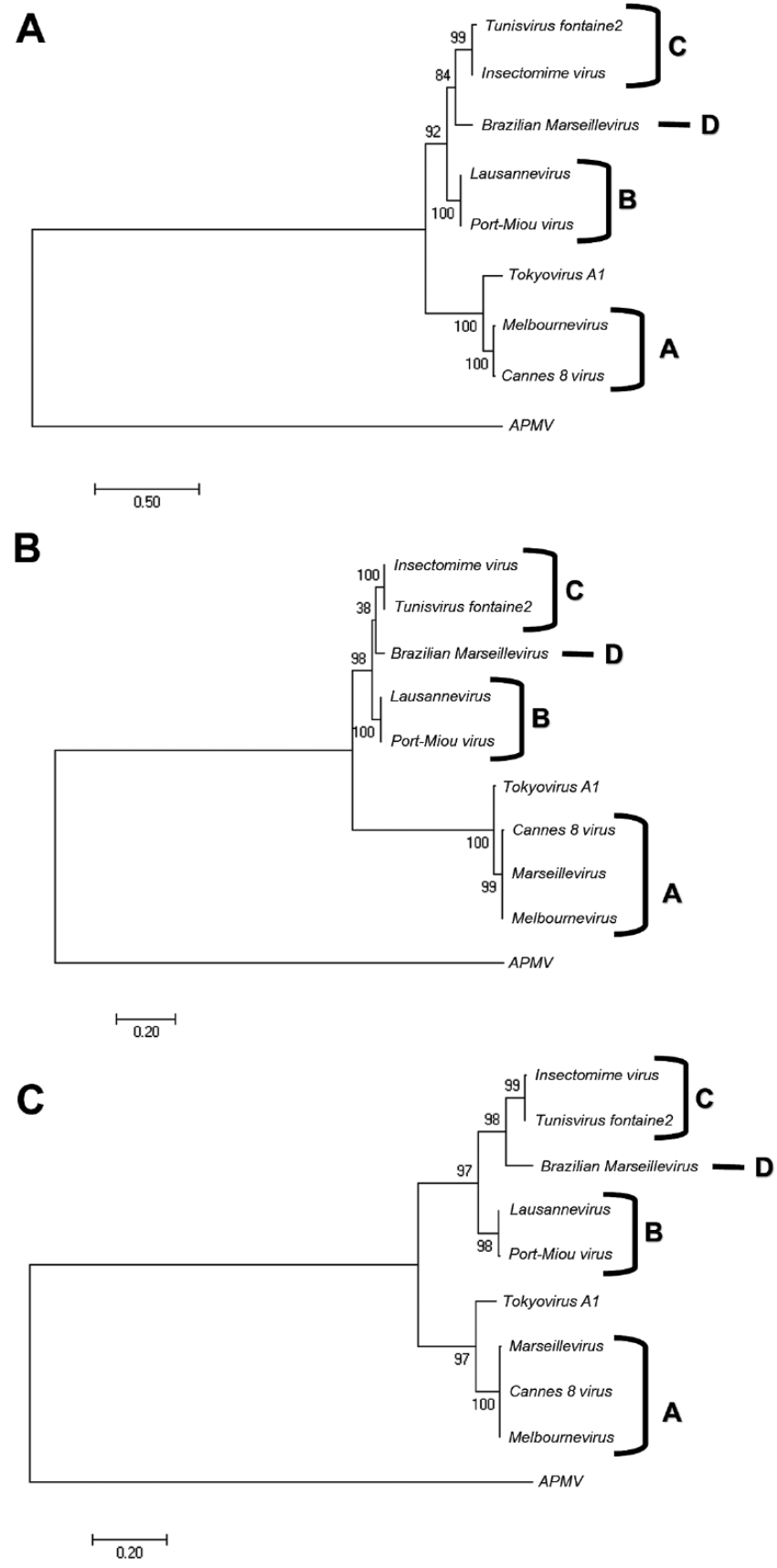

Fig. 5. Unrooted maximum-likelihood phylogenetic trees of B-family DNA polymerase (A), PCNA (B), and DNA-directed RNA polymerase alpha subunit (C) sequences constructed using MEGA7 software (26). The trees were reconstructed based on alignments (DNA polymerase, 1,103 sites; PCNA, 257 sites; RNA polymerase alpha subunit, 452 sites) derived from the full-length alignment in which any column containing a gap was discarded. Numbers at the branch points denote percent bootstrap values. The accession numbers of respective sequences are listed in Tables S1 S3. The letters A, B, C, and D indicate the subclades of Marseilleviridae.

technical assistances with the TEM analysis, and Ryuta Kasatani, Shingo Murono, and Tatsuya Mikami from my laboratory for their technical assistance and useful discussions. I would also like to thank Drs. Hiroyuki Ogata of Kyoto University, Hiroyuki Yamaguchi of Hokkaido University, Satoru Arai of National Institute of Infectious Diseases of Japan, Bernald La Scola of Faculté de Médecine, AixMarseille Université, and Chantal Abergel of CNRS of Aix-Marseille Université, for their useful comments and discussions. 


\section{References}

1. Abergel, C., M. Legendre, and J.-M. Claverie. 2015. The rapidly expanding universe of giant viruses: Mimivirus, Pandoravirus, Pithovirus and Mollivirus. FEMS Microbiol. Rev. 39:779-796.

2. Aherfi, S., I. Pagnier, G. Fournous, D. Raoult, B. La Scola, and P. Colson. 2013. Complete genome sequence of Cannes 8 virus, a new member of the proposed family "Marseilleviridae." Virus Genes 47:550-555.

3. Aherfi, S., M. Boughalmi, I. Pagnier, G. Fournous, B. La Scola, D. Raoult, and P. Colson. 2014. Complete genome sequence of Tunisvirus, a new member of the proposed family Marseilleviridae. Arch. Virol. 159:2349-2358.

4. Aherfi, S., P. Colson, B. La Scola, and D. Raoult. 2016. Giant viruses of amoebas: an update. Front. Microbiol. doi:10.3389/fmicb.2016.00349.

5. Arantes, T.S., R.A.L. Rodrigues, L.K.S. Silva, et al. 2016. The large Marseillevirus explores different entry pathways by forming giant infectious vesicles. J. Virol. 90:5246-5255.

6. Arslan, D., M. Legendre, V. Seltzer, C. Abergel, and J.-M. Claverie. 2011. Distant Mimivirus relative with a large genome highlights the fundamental features of Megaviridae. Proc. Natl. Acad. Sci. U.S.A. 108:17486-17491.

7. Boughalmi, M., I. Pagnier, S. Aherfi, P. Colson, D. Raoult, and B. La Scola. 2013. First isolation of a Marseillevirus in the diptera syrphidae Eristalis tenax. Intervirol. 56:386-394.

8. Boyer, M., N. Yutin, I. Pagnier, et al. 2009. Giant Marseillevirus highlights the role of amoebae as a melting pot in emergence of chimeric microorganisms. Proc. Natl. Acad. Sci. U.S.A. 106:2184821853.

9. Colson, P., N. Yutin, S.A. Shabalina, C. Robert, G. Fournous, B. La Scola, D. Raoult, and E.V. Koonin. 2011. Viruses with more than 1,000 genes: Mamavirus, a new Acanthamoeba polyphaga mimivirus strain, and reannotation of Mimivirus genes. Genome Biol. Evol. 3:737-742.

10. Colson, P., I. Pagnier, N. Yoosuf, G. Fournous, B. La Scola, and D. Raoult. 2013. "Marseilleviridae", a new family of giant viruses infecting amoebae. Arch. Virol. 158:915-920.

11. Dornas, F.P., F.L. Assis, S. Aherfi, T. Arantes, J.S. Abrahão, C. Philippe, and B. La Scola. 2016. A Brazilian Marseillevirus is the founding member of a lineage in family Marseilleviridae. Viruses 8: doi:10.3390/v8030076.

12. Doutre, G., N. Philippe, C. Abergel, and J.-M. Claverie. 2014. Genome analysis of the first Marseilleviridae representative from Australia indicates that most of its genes contribute to virus fitness. J. Virol. 88:14340-14349.

13. Doutre, G., B. Arfib, P. Rochette, J.-M. Claverie, P. Bonin, and C. Abergel. 2015. Complete genome sequence of a new member of the Marseilleviridae recovered from the Brackish submarine spring in the Cassis Port-Miou Calanque, France. Genome Announc. 3:e1148-15.

14. Fischer, M.G., M.J. Allen, W.H. Wilson, and C.A. Suttle. 2010. Giant virus with a remarkable complement of genes infects marine zooplankton. Proc. Natl. Acad. Sci. U.S.A. 107:19508-19513.
15. La Scola, B., S. Audic, C. Robert, L. Jungang, X. de Lamballerie, M. Drancourt, R. Birtles, J.-M. Claverie, and D. Raoult. 2003. A giant virus in amoebae. Science 299:2033.

16. Lagier, J.-C., F. Armougom, M. Million, et al. 2012. Microbial culturomics: paradigm shift in the human gut microbiome study. Clin. Microbiol. Infect. 18:1185-1193.

17. Legendre, M., J. Bartoli, L. Shmakova, et al. 2014. Thirty-thousandyear-old distant relative of giant icosahedral DNA viruses with a pandoravirus morphology. Proc. Natl. Acad. Sci. U.S.A. 111:42744279 .

18. Legendre, M., A. Lartigue, L. Bertaux, et al. 2015. In-depth study of Mollivirus sibericum, a new 30,000-y-old giant virus infecting Acanthamoeba. Proc. Natl. Acad. Sci. U.S.A. 112:E5327-E5335.

19. Phan, T.G., C. Desnues, W.M. Switzer, C.E. Djoko, B.S. Schneider, X. Deng, and E. Delwart. 2015. Absence of giant blood Marseille-like virus DNA detection by polymerase chain reaction in plasma from health US blood donors and serum from multiply transfused patients from Cameroon. Transfusion 55:1256-1262.

20. Philippe, N., M. Legendre, G. Doutre, et al. 2013. Pandoraviruses: amoeba viruses with genomes up to $2.5 \mathrm{Mb}$ reaching that of parasitic eukaryotes. Science 341:281-286.

21. Popgeorgiev, N., M. Boyer, L. Fancello, et al. 2013. Marseilleviruslike virus recovered from blood donated by asymptomatic humans. J. Infect. Dis. 208:1042-1050.

22. Raoult, D., S. Audic, C. Robert, C. Abergel, P. Renesto, H. Ogata, B. La Scola, M. Suzan, and J.-M. Claverie. 2004. The 1.2-megabase genome sequence of Mimivirus. Science 306:1344-1350.

23. Reteno, D.G., S. Benamar, J.B. Khalil, J. Andreani, N. Armstrong, T. Klose, M. Rossmann, P. Colson, D. Raoult, and B. La Scola. 2016. Faustovirus, an Asfarvirus-related new lineage of giant viruses infecting amoebae. J. Virol. 89:6585-6594.

24. Takemura, M., S. Yokobori, and H. Ogata. 2015. Evolution of eukaryotic DNA polymerases via interaction between cells and large DNA viruses. J. Mol. Evol. 81:24-33.

25. Takemura, M. 2016. The draft genome sequence of Tokyovirus, a member of Marseilleviridae isolated from the Arakawa River of Tokyo, Japan. Genome Announc. 4:e00429-16.

26. Tamura, K., G. Stecher, D. Peterson, A. Filipski, and S. Kumar. 2013. MEGA6: molecular evolutionary genetics analysis ver. 6.0. Mol. Biol. Evol. 30:2725-2729.

27. Thomas, V., C. Bertelli, F. Collyn, N. Casson, A. Telenti, A. Goesmann, A. Croxatto, and G. Greub. 2011. Lausannevirus, a giant amoebal virus encoding histone doublets. Environment. Microbiol. 13:1454-1466.

28. Yoosuf, N., N. Yutin, P. Colson, et al. 2012. Related giant viruses in distant locations and different habitats: Acanthamoeba polyphaga moumouvirus represents a third lineage of the Mimiviridae that is close to the Megavirus lineage. Genome Biol. Evol. 4:1324-1330. 